\title{
Quantification of myocardial blood flow will reform the detection of CAD
}

\author{
Juhani Knuuti, MD, Sami Kajander, MD, Maija Mäki, MD, \\ and Heikki Ukkonen, MD
}

\section{INTRODUCTION}

The detection of functional consequences of epicardial coronary artery disease (CAD) has established role in guiding the therapy of the disease. ${ }^{1}$ In addition, the assessment of impairment in microcirculatory reactivity has recently gained more interest. Estimates of myocardial perfusion contain independent prognostic information about future major cardiac events and perfusion assessment is also useful in the monitoring of the effectiveness of risk reduction strategies. ${ }^{1}$

The standard assessment of myocardial perfusion is based on its relative distribution. This approach has obvious limitations since the interpretation is based on the assumption that the best perfused region is normal and can be used as a reference. Using quantification this limitation can be avoided and using absolute parameters instead of relative ones is expected to provide benefits in several clinical scenarios. Despite the recognized potential, the clinical use of absolute quantification has remained scarce.

Recently, several imaging techniques have been studied aiming for the quantitative measurement of perfusion. In addition nuclear imaging, magnetic resonance imaging, and echocardiography have been investigated and shown promising preliminary quantitative results. ${ }^{2,3}$ Currently, the most robust technique to quantify perfusion noninvasively in human heart is positron emission tomography (PET). However, although the use of PET in cardiac imaging is rapidly increasing, the image interpretation has still been chiefly based on relative distribution of perfusion. ${ }^{4}$

This review is aiming for brief summary of the current knowledge of quantification of myocardial perfusion for the detection of clinical CAD. The aim is to demonstrate the

From the Turku PET Centre, University of Turku, Turku, Finland. This work was supported by the Finnish Centre of Excellence on Molecular Imaging in Cardiovascular and Metabolic Research.

Received for publication May 5, 2009; final revision accepted May 6, 2009.

Reprint requests: Juhani Knuuti, MD, Turku PET Centre, University of Turku, Kiinamyllynkatu 4-8, 20520 Turku, Finland; juhani.knuuti@ tyks.fi; juhani.knuuti@utu.fi.

J Nucl Cardiol 2009;16:497-506.

$1071-3581 / \$ 34.00$

Copyright $(2009$ by the American Society of Nuclear Cardiology. doi:10.1007/s12350-009-9101-1 potential of quantification using several real clinical examples where the additional information gained from quantification has significant impact on the clinical findings.

\section{THE TECHNIQUES OF MYOCARDIAL PERFUSION QUANTIFICATION}

There are two major determinants that define the potential of imaging technique for quantification of myocardial perfusion. The imaging device and its performance will define how accurate and robust is the measurement of the signal that need to be quantified. The other main factor is the nature of the tracer or the contrast agent. The detailed analysis of different imaging technologies and their performance is beyond the scope of this review.

PET is by nature a quantitative technique and currently the most robust technique to measure perfusion noninvasively in human heart. PET allows very fast and effective imaging protocols with low radiation burden. ${ }^{5-8}$

Several tracers have been used for the measurement of myocardial perfusion with PET, in particular, ${ }^{15} \mathrm{O}$-water, $\left.{ }^{9}\right] \quad{ }^{13} \mathrm{~N}$-ammonia, ${ }^{10}$ the potassium analog ${ }^{82} \mathrm{Rb}^{11}$ Recently also new ${ }^{18}$ Fluoride-labeled ${ }^{12,13}$ as well as ${ }^{68} \mathrm{Ga}$-labeled compounds have been tested. ${ }^{14}$

Currently, ${ }^{15} \mathrm{O}$-water and ${ }^{13} \mathrm{~N}$-ammonia are the tracers most widely used for the quantification of myocardial perfusion with PET. Tracer kinetic models for quantification of perfusion have been successfully validated in animals against the radiolabeled microsphere method over a wide flow range for both tracers. ${ }^{9,10}$

Single-tissue compartment models for an inert freely diffusible tracer are used for calculation of MBF by use of ${ }^{15} \mathrm{O}$-water. ${ }^{9}$ For ${ }^{13} \mathrm{~N}$-ammonia a three-compartment model taking into account the myocardial metabolic trapping and whole-body metabolism has been used for calculation of perfusion. ${ }^{10,15}$ Typically, the models also correct for the partial volume effect and spillover from the left ventricular chamber into the myocardial regions. These two tracers have been found to be equivalent for quantifying myocardial perfusion. ${ }^{16}$

There are, however, some obvious differences between these two tracers. ${ }^{13} \mathrm{~N}$-ammonia is extracted from blood with an extraction fraction lower than $100 \%$ and the extraction is inversely related to the perfusion (Figure 1). When ${ }^{15} \mathrm{O}$-water is used, perfusion is 


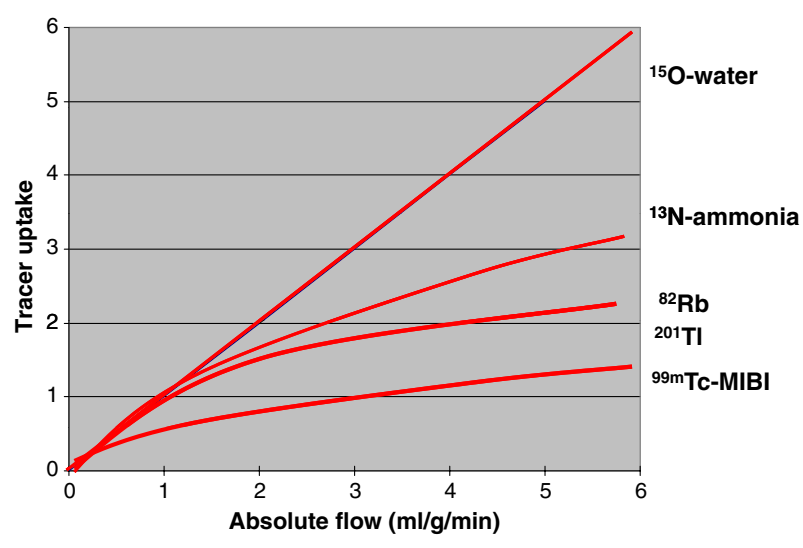

Figure 1. Graphical presentation of the relationship between absolute myocardial perfusion and tracer uptake. The lines are only rough estimates of the tracer uptake characteristics. However, in all tracers except 150-water, the tracer extraction is reduced when perfusion is increased leading to underestimation of perfusion if only tracer uptake is used.

estimated from the tracer's washout from the myocardium, whereas with ${ }^{13} \mathrm{~N}$-ammonia, perfusion is calculated from the tracer's uptake by myocardium.

The most important limitation of perfusion tracers as regard to quantification is the suboptimal myocardial extraction of the tracer. In addition to suboptimal extraction level, even more problematic phenomenon is that the extraction is reduced with increasing perfusion (Figure 1). When extraction fraction is reduced with increasing perfusion the uptake of tracer is not linear with perfusion and higher flows are underestimated. This must be corrected in the mathematical model by increasing the higher range perfusion values using specific equations. However, in the case the extraction is much blunted very high correction factors need to be used or the correction may become impossible. It needs to be noted also that high correction factors also multiplies the noise of the measurements leading to larger scatter of the values with high perfusion rates.

Although kinetic models have been proposed for quantification of perfusion by use of ${ }^{82} \mathrm{Rb}$, all these models are limited by the dependence of the myocardial extraction fraction on the perfusion rate. The recent animal validation studies with ${ }^{82} \mathrm{Rb}$ has shown reasonable good linearity but as expected the scatter in high flow rates is quite high. ${ }^{11,17}$ This does not necessarily mean that ${ }^{82} \mathrm{Rb}$ cannot be used to quantify myocardial perfusion in clinical situations but may indicate that different cut-off values for normal and abnormal perfusion need to be used or that the accuracy may not be as good than with other perfusion tracers.

It needs to be noted, however, that this phenomenon is very evident also with other tracers such as SPECT perfusion tracers (Figure 1), MRI, and CT contract agents.

\section{QUANTITATIVE PERFUSION IMAGING PROTOCOLS}

The perfusion imaging protocol depends on which tracer is used. With PET imaging the stress study is performed using pharmacologic stressors such as adenosine, dipyridamole, or dobutamine. The dynamic imaging protocols have been standardized. ${ }^{1,18}$ With PET tracers such as ${ }^{82} \mathrm{Rb}$ and ${ }^{15} \mathrm{O}$-water studies (half-lives 76 seconds and 112 seconds) the stress study can be performed practically without delay after the rest study. With ${ }^{13} \mathrm{~N}$-ammonia stress testing is delayed for about $30 \mathrm{~min}$ utes to allow tracer decay. If method to correct patient motion between stress and rest studies is not available, second low-dose attenuation is needed. In all studies quality control process is needed to ensure optimal alignment of the attenuation and PET emission scans and, if necessary, misalignment needs to be corrected. ${ }^{19,20}$

If the system is capable to list mode acquisition, the data can be collected as ECG gated mode that allows the simultaneous assessment of regional and global left ventricular wall motion from the same scan data. This is particularly practical when ${ }^{82} \mathrm{Rb}$ is used as tracer. The total time required for whole study session depends on the tracer used. With ${ }^{15} \mathrm{O}$-water and ${ }^{82} \mathrm{Rb}$ the whole session can be finished in 30 minutes and with ${ }^{13} \mathrm{~N}$-ammonia in 80 minutes. The protocols can be further shortened significantly since in hybrid approaches only single stress perfusion imaging may be needed especially when using quantification ${ }^{21}$ and the protocols with all tracers can be as short as 15 minutes.

\section{INTERPRETATION OF ABSOLUTE MYOCARDIAL PERFUSION RESULTS}

When quantitative results are obtained, the criteria for interpretation must be redefined. As regard to perfusion reserve typically values above 2.5-2.7 have been regarded as normal. ${ }^{5}$

As regard to absolute perfusion in $\mathrm{mL} / \mathrm{g} /$ minute, a little clinical evidence is available and it also appears that different traces may provide different cutoffs..$^{15,21-23}$ In the study by Muzik et $\mathrm{al}^{5}$ using ${ }^{13} \mathrm{~N}$-ammonia as tracer, the optimal cutoff value was found to be quite low $(1.52 \mathrm{~mL} / \mathrm{g} /$ minute $)$ to separate ischemic from noninschemic regions. In our recent analysis using ${ }^{15} \mathrm{O}$-water the ideal cutoff for absolute stress perfusion was $2.5 \mathrm{~mL} / \mathrm{g} /$ minute, the range from 2.0 to 2.5 being mildly reduced. ${ }^{23}$ Naturally the more severe is ischemia the lower is the stress perfusion and in clearly ischemic regions the stress perfusion is just above resting values $(1.0-2.0 \mathrm{~mL} / \mathrm{g} /$ minute $)$.

With ${ }^{82} \mathrm{Rb}$ very limited clinical data is available. In the study by Anagnostopoulos et $\mathrm{al}^{22}$ linearity with the 
perfusion or perfusion reserve versus coronary diameter stenosis was detected but the cutoffs for perfusion reserve and absolute stress perfusion values seem to be lower than the numbers reported for ${ }^{13} \mathrm{~N}$-ammonia or ${ }^{15} \mathrm{O}$-water earlier. More studies are warranted.

One of the main issues which need to be kept in mind is that the interpretation of the quantified parametric perfusion images is different from the traditional images. In the traditional tracer distribution images the upper range of perfusion is seen blunted so that at high perfusion range very little changes in the tracer uptake is seen (Figure 2). This is mainly caused by the decreasing tracer extraction fraction at high perfusion range in most tracers. When quantification is performed, the perfusion scale will also be expanded to high flows. This leads to apparently more heterogeneous perfusion images since the upper range 'smoothing' is uncovered. This has been known for long with ${ }^{15} \mathrm{O}$-water which does not produce qualitative perfusion images at all but only quantitative results. The same phenomenon is likely to happen automatically with the new tracers with high extraction fraction ${ }^{12,13}$ and also with other tracers after quantification. This means that the traditional criteria for ischemic regions in the images need to be redefined when quantification is applied.

It needs to be acknowledged that there is still limited clinical data about the optimal cut-off perfusion reserve or stress perfusion values to distinguish ischemic from nonischemic regions. Furthermore, display of quantified parametric perfusion images needs to be standardized. One option used in the clinical examples of this article is to display the images using fixed scale so that each color or gray zone represents specific absolute number in quantification. All examples shown in this article have been displayed in this way by choosing the maximum value as $3.5 \mathrm{~mL} / \mathrm{g} /$ minute.

\section{CLINICAL SCENARIOS WHERE QUANTIFICATION IS BENEFICIAL}

The incremental value of quantitative analysis for the clinical decision making has been sparsely studied. In those few studies it was found that the accuracy was further improved by quantitative analysis. ${ }^{5-7,21}$ Based on the currently available information there are several clinical scenarios where the quantification is likely changing the clinical interpretation.

\section{Multivessel CAD}

The typical situation where quantification will provide incremental information is patient with multivessel disease. Relative assessment of perfusion distribution often uncovers only the coronary territory supplied by the most severe stenoses (Figure 3). In multivessel disease coronary flow reserve may be variably abnormal in all territories thereby reducing the heterogeneity of perfusion between "normal",

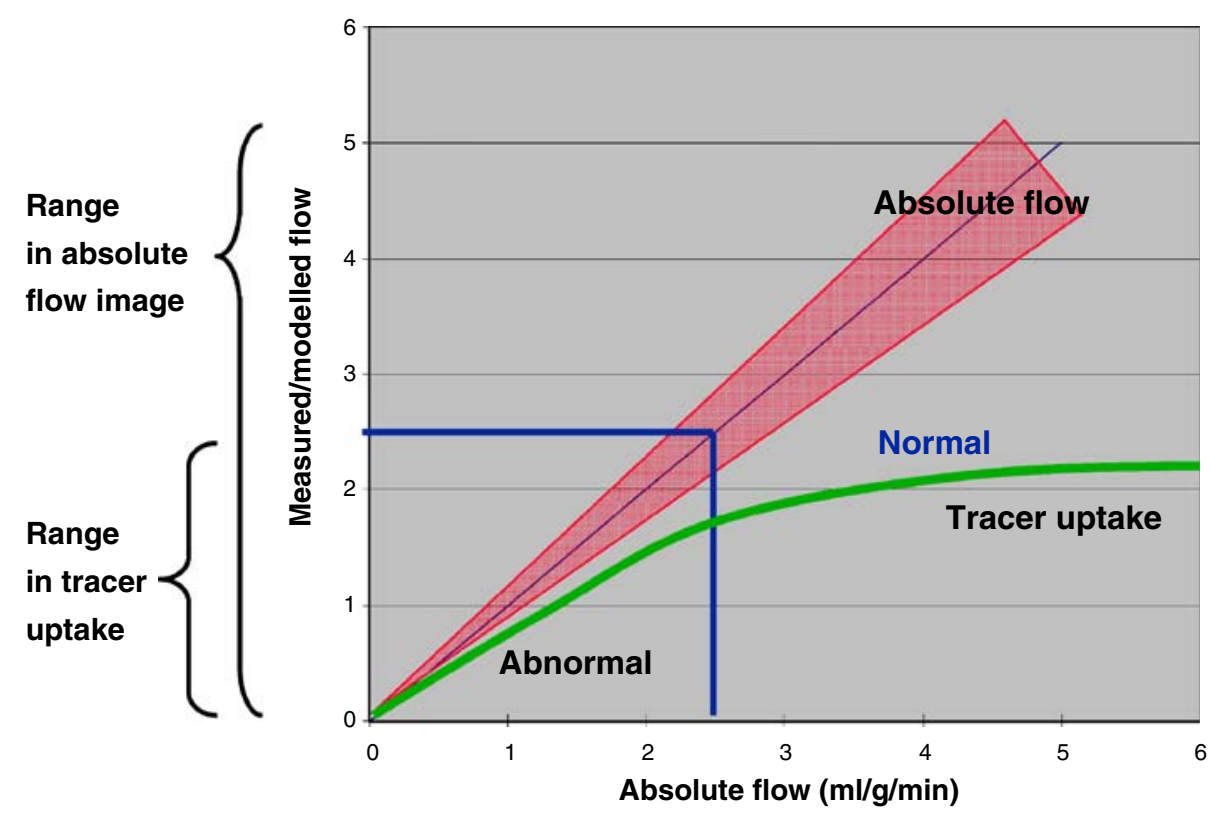

Figure 2. Theoretical presentation of the relationship between absolute myocardial perfusion and measured perfusion after quantification. The mathematical modeling is aiming to correct the nonlinear relationship between the perfusion and tracer uptake. The resulted quantified perfusion values will have different scale than the original relative perfusion images. Due to correction, however, the noise in high perfusion range will be increased. 

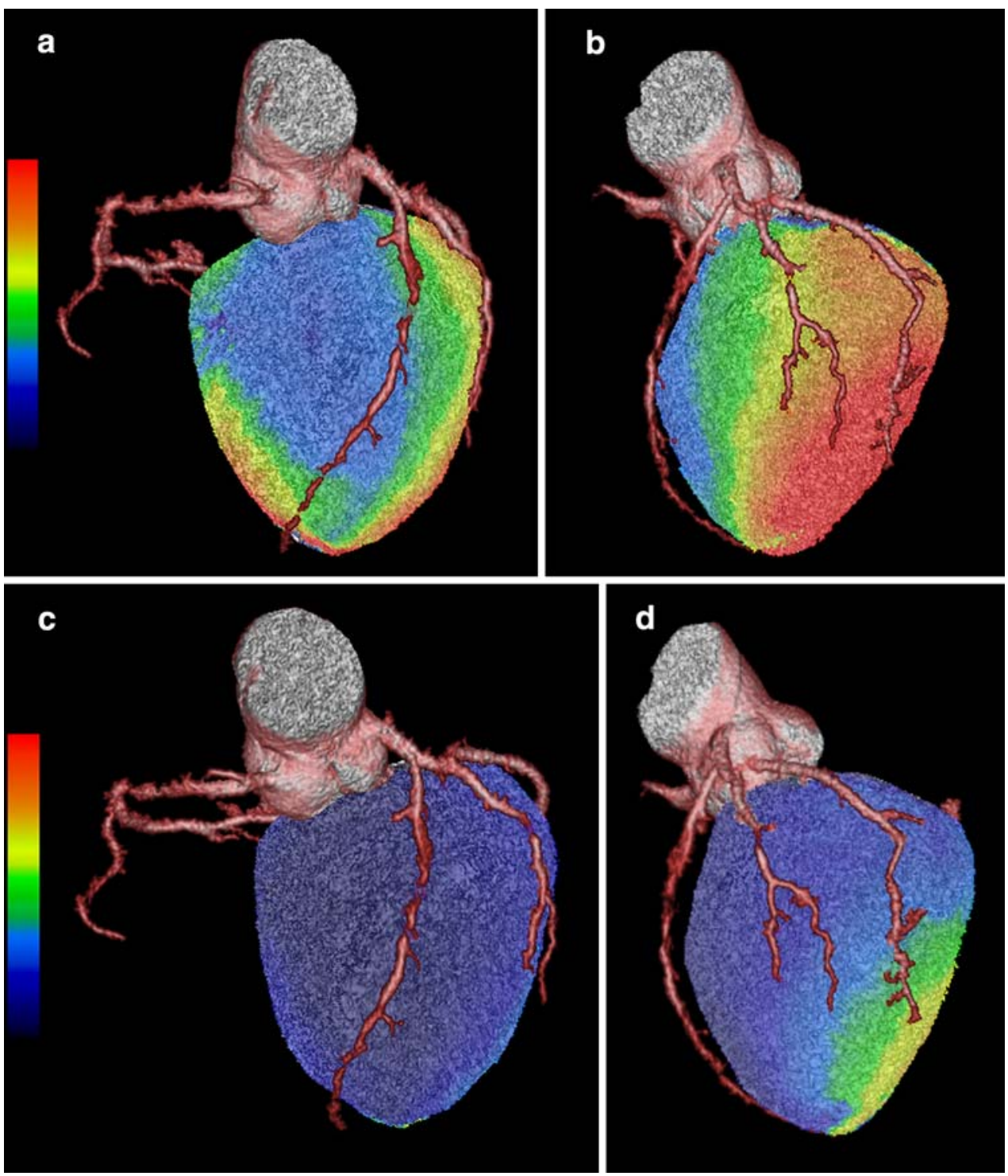

Figure 3. In situations of complex multivessel CAD quantification provides added value in the assessment of severity of ischemia. Three-dimensional cardiac hybrid PET-CT images providing a panoramic view of parametric ${ }^{15} \mathrm{O}$-water stress perfusion and $\mathrm{CT}$ angiography in patient with effort angina. The images ( $\mathbf{A}$ and $\mathbf{B}$ ) scaled to relative scale where the best perfused region is set to maximum and has the brightest color (in rainbow scale lowest $=$ blue and highest $=$ red). The hybrid images of anterior (A) and lateral (B) views suggested large perfusion defect in the anterior wall but other areas were considered normally perfused. However, in the images scaled according to absolute scale $(\mathbf{C}$ and $\mathbf{D})(0 \mathrm{~mL} / \mathrm{g} / \mathrm{min}=$ blue and $3.5 \mathrm{~mL} / \mathrm{g} / \mathrm{min}=$ red $)$ uncovered reduction of perfusion also in lateral wall supplied by left circumflex artery. The normal perfusion was found only in inferoapical wall. The finding of multi-vessel disease was confirmed also in invasive angiography.

and "abnormal" zones. This may be particularly helpful in patients with multiple perfusion abnormalities (Figure 4).

This problem can be solved immediately by quantification since it provides independent information about all myocardial territories. ${ }^{5-7,21}$ Also determination of the response of left ventricular ejection fraction to stress from gated perfusion data will help. ${ }^{24}$

\section{Balanced Multivessel CAD}

Quantification is obviously helpful in patients with diffuse CAD or balanced disease where relative assessment of myocardial perfusion cannot uncover global reduction in perfusion (Figure 5). Typically, in relative analysis of perfusion the images look homogenous and 

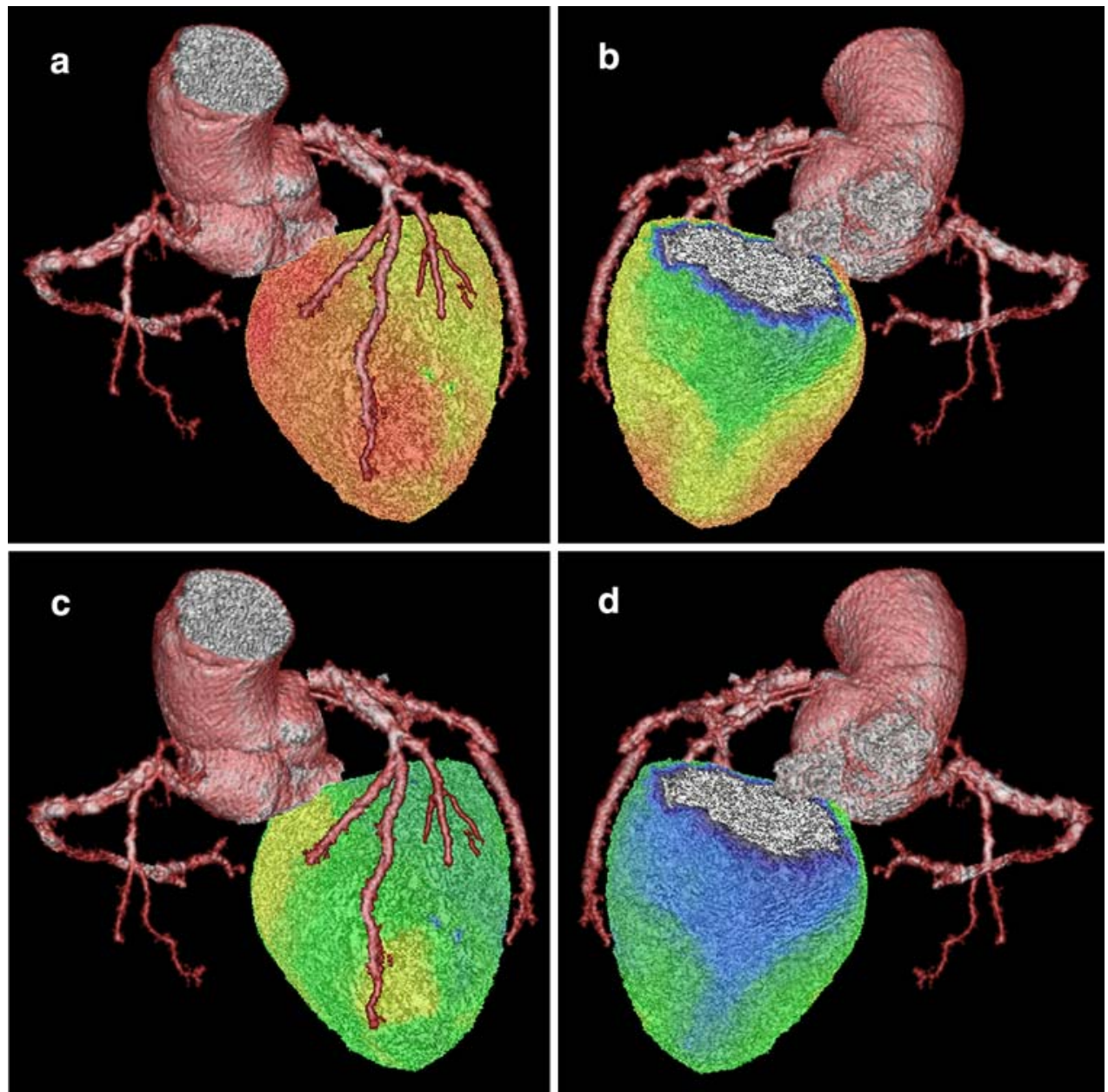

Figure 4. Three-dimensional cardiac hybrid PET-CT images of patient with effort angina with similar technique than used in Figure 3. The images (A and B) scaled to relative scale of anterior (A) and posterior (B) views suggested small perfusion defect in the posterior wall but other areas were considered normally perfused. However, in the images scaled according to absolute scale (C and D) abnormal perfusion was found also in the anterior and lateral walls. The finding was confirmed in invasive angiography.

the disease can be completely missed if no other signs of multivessel disease are evident.

Earlier this problem has been regarded as clinically uncommon but in recent preliminary analysis $5-10 \%$ of patients with suspected CAD will display balanced CAD pattern. ${ }^{21}$

\section{The Exclusion of CAD in Symptomatic Patients}

Although the normalcy rate of perfusion imaging has been reported to be quite high (around 90\%) subtle irregularities are commonly classified as equivocal findings. In addition, in recent clinical studies the specificity of perfusion imaging has been quite low $(50-70 \%)$ but sensitivity has remained high. Although, this phenomenon can be partly explained by referral bias, part of the explanation lays on the interpretation of subtle perfusion irregularities as suspicious findings.

In quantitative analysis the relative heterogeneity has less impact on the interpretation since high absolute perfusion in a region can be regarded as normal despite the inhomogeneous perfusion distribution (Figure 6). Inversely, the use of quantification makes it more confident to interpret also subtle perfusion irregularities as abnormal when absolute perfusion is low.

It has been also documented that PET is more accurate than SPECT in traditionally difficult patient populations such as obese and diabetic subjects ${ }^{25}$ and this phenomenon may be linked with the more quantitative nature of PET imaging. 

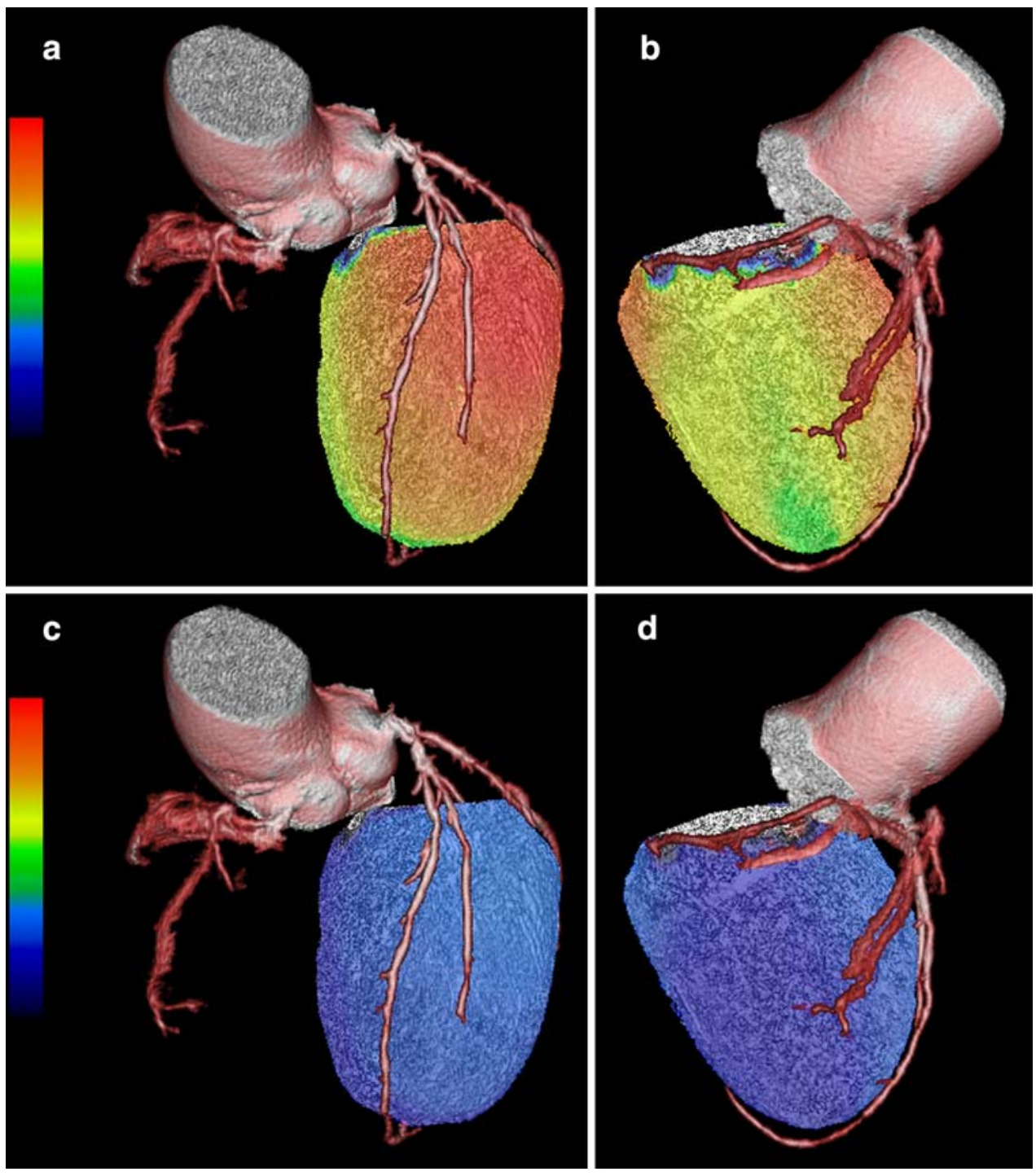

Figure 5. Three-dimensional cardiac hybrid PET-CT images of patient with effort angina with similar technique than used in Figure 3. The images (A and B) scaled to relative scale of anterior (A) and posterior (B) views suggested only small suspicious perfusion defect in the inferoapical wall. However, in the images scaled according to absolute scale (C and $\mathbf{D})$ global reduction in all myocardial regions was detected suggesting balanced 3 vessel CAD. The finding was confirmed in $\mathrm{CT}$ and invasive angiography.

\section{Patients with Microvascular Disease}

The patients with microvascular disease are characterized by diffuse reduction in myocardial perfusion (Figure 7). This finding cannot be exposed by standard relative perfusion analysis. Also the early coronary dysfunction liked with early CAD can be detected only with quantitative analysis of perfusion.

\section{Revascularized Patients}

Patients who have undergone coronary bypass revascularization have typically very complex CAD with advanced disease in native vessels and potential graft problems. In these patients the relative perfusion patterns may be very atypical. With quantitative analysis of perfusion the confidence of reading is likely improved. In this complex situation also hybrid imaging may be very useful. (Figure 8).

\section{LIMITATIONS OF PERFUSION QUANTIFICATION}

Although quantification of myocardial perfusion has obviously a great clinical potential there are several limitations that need to be recognized. 

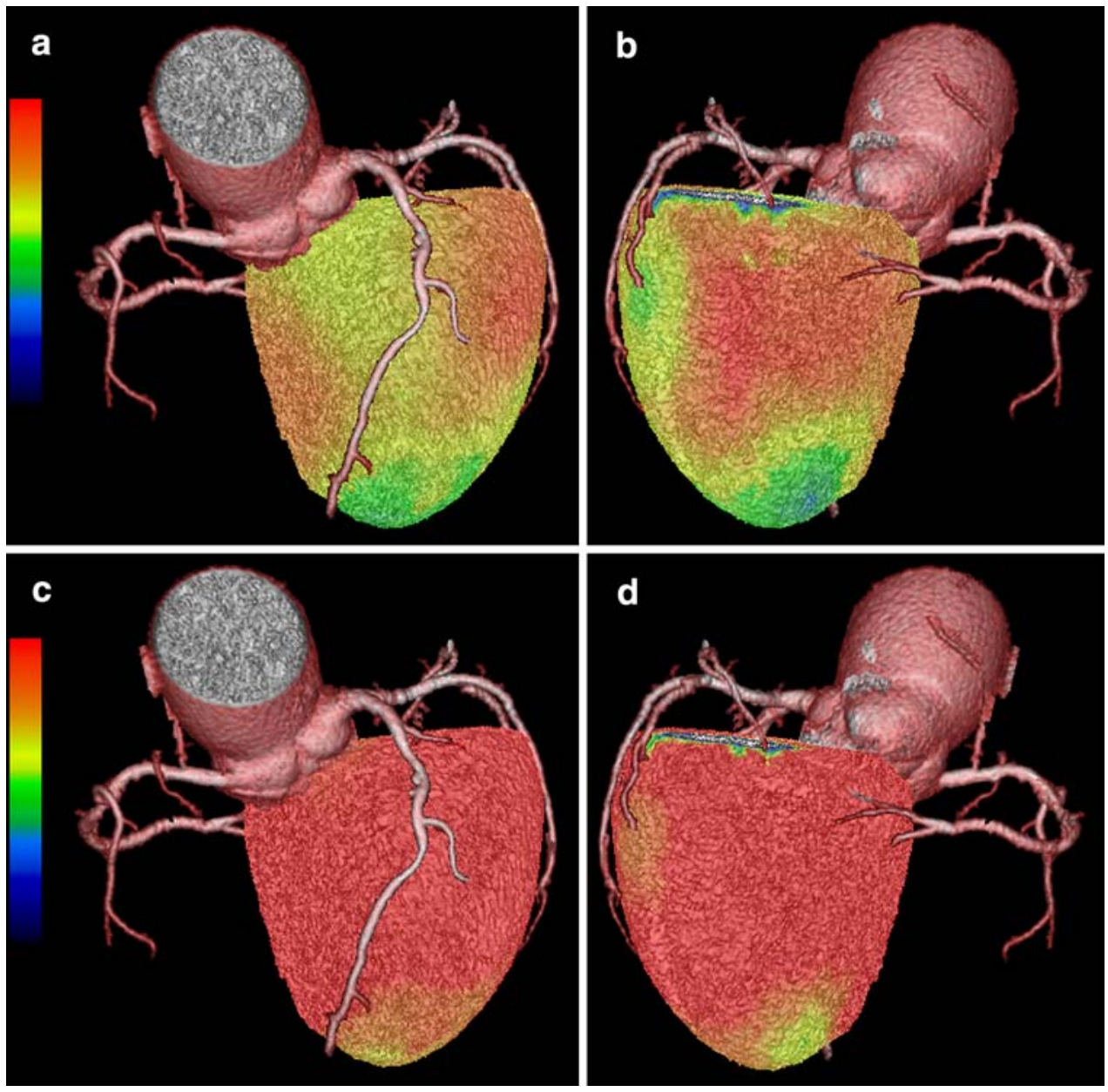

Figure 6. Three-dimensional cardiac hybrid PET-CT images of patient with effort angina with similar technique than used in Figure 3. The images (A and B) scaled to relative scale of anterior (A) and posterior (B) views suggested several suspicious perfusion defects in the apical and lateral walls. However, in the images scaled according to absolute scale $(\mathbf{C}$ and $\mathbf{D})$ perfusion was high in all myocardial regions. This was in agreement with angiography where only small nonsignificant coronary lesions were detected.

The knowledge of optimal cut-off values for absolute perfusion needs to be studied in larger populations and in various tracers. Furthermore, the quantification process using each tracer and the interpretation needs to be standardized. More information is needed about different subpopulations such as diabetic and obese patients, patients with heart failure, and revascularized patients.

Even with optimized techniques it remains the fact that perfusion imaging does not provide morphological information. The perfusion imaging only provides information about existence and severity of perfusion abnormalities but not about the mechanism, e.g. the patients with diffuse balanced coronary heart disease and those with microvascular dysfunction leading to globally compromised perfusion cannot be separated by perfusion imaging. Therefore, the combined or hybrid imaging with CT angiography is believed to provide incremental information in patients with suspected $\mathrm{CAD}^{26}$

Myocardial perfusion imaging provides a simple and accurate integrated measure of the effect of all parameters on coronary resistance and tissue perfusion, but at the same time it cannot dissect the effect of specific coronary plaque in the case of serial changes or when combined with microvascular disease (Figure 7).

A special group of patients where perfusion is difficult to interpret are patients with heart failure. In heart failure absolute perfusion and perfusion are commonly reduced and also heterogeneous. This makes the interpretation without anatomical information very challenging. ${ }^{27}$ 

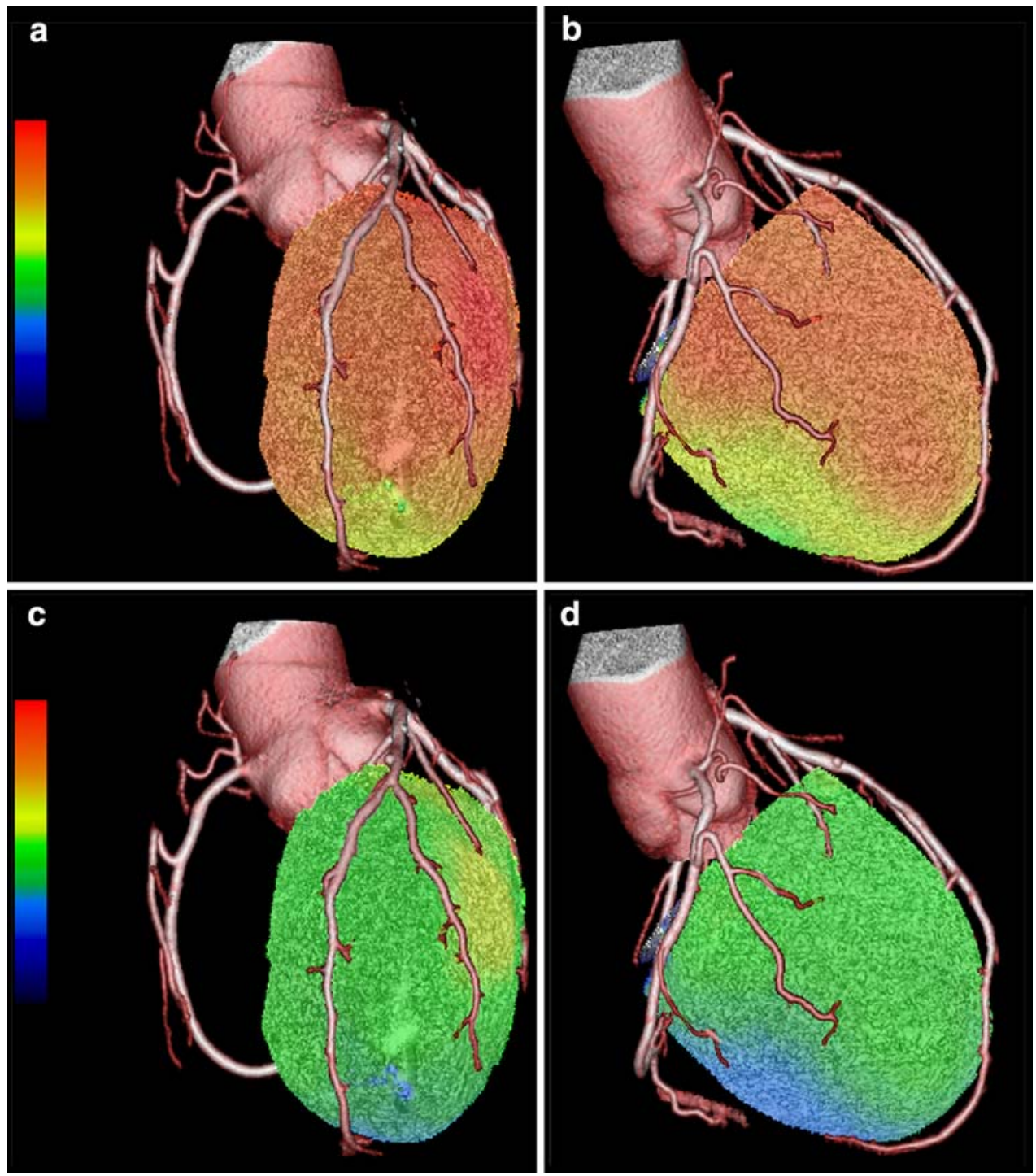

Figure 7. Three-dimensional cardiac hybrid PET-CT images of patient with angina with similar technique than used in Figure 3. The images (A and $\mathbf{B}$ ) scaled to relative scale of anterior (A) and right (B) views. No obvious perfusion abnormalities were detected in the relative scale images. In the images scaled according to absolute scale $(\mathbf{C}$ and $\mathbf{D})$ perfusion was heterogeneously abnormally low in most myocardial regions. In CT angiography and invasive angiography only minor epicardial coronary lesions were detected. The most probable explanation for abnormal perfusion in this patient is microvascular disease.

\section{SUMMARY AND FUTURE DEVELOPMENTS}

Quantification of myocardial perfusion will likely become standard technique also in clinical studies while more information and clinical evidence becomes available. Even the current limited experience demonstrates potential benefits in several patient groups.

To become standard technique the access to tracers that enable accurate quantification needs to be improved. Whether quantification of ${ }^{82} \mathrm{Rb}$ kinetics will be accurate enough for clinical use remains to be seen. New
${ }^{18}$ Fluoride-labeled tracers with high extraction fraction are very promising.

The concept of hybrid imaging to deliver comprehensive integrated morphological and functional information is particularly appealing. ${ }^{26}$ In addition to being intuitively convincing, these images provide a panoramic view of the myocardium, the regional myocardial perfusion, or viability and the coronary artery tree, thus eliminating uncertainties in the relationship of perfusion defects, scar regions, and diseased coronary arteries. 

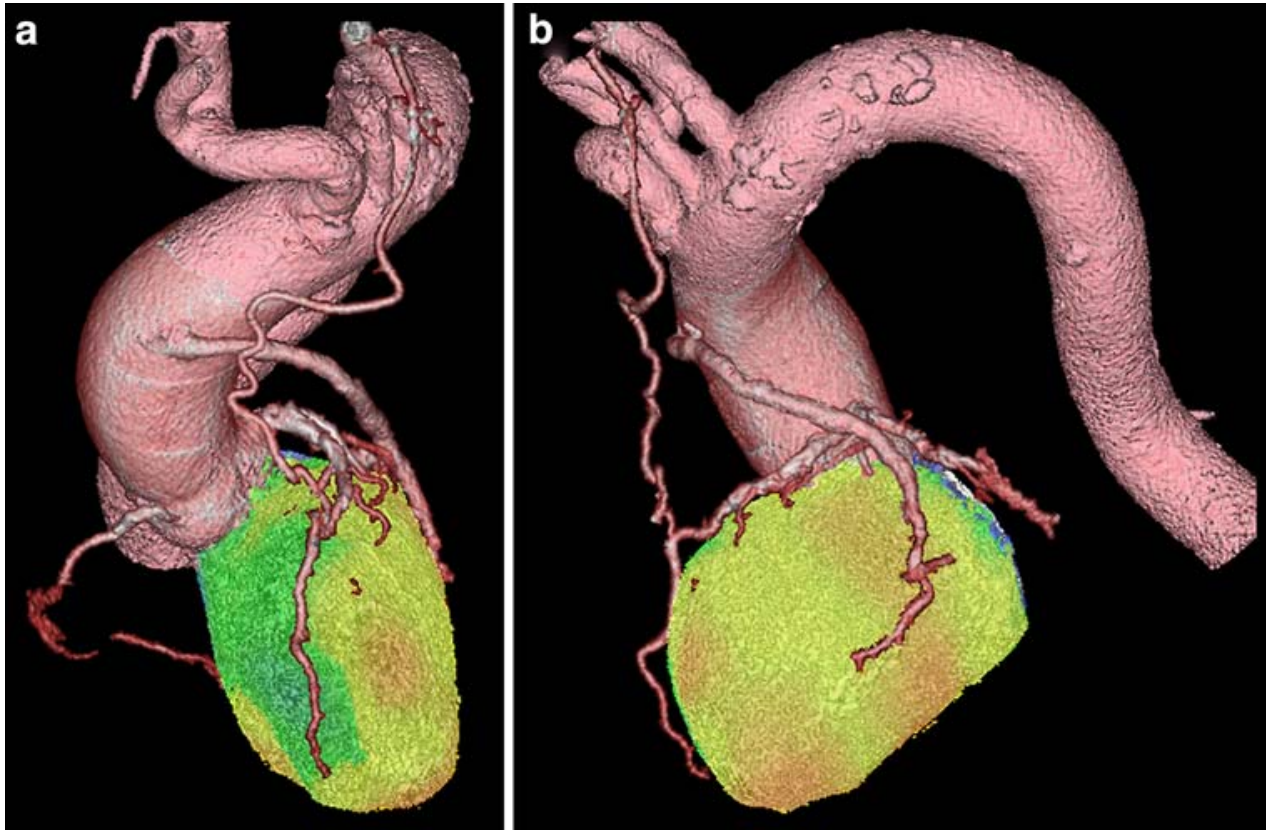

Figure 8. In situations of complex coronary CAD and after bypass surgery quantification with hybrid cardiac imaging provides added value. CT coronary angiography may allow visualization of patent bypass grafts but the evaluation of the anastomoses remains difficult. A conclusion about hemodynamic relevant lesions can only be reached in conjunction with perfusion. The image documents residual ischemia in the left anterior descending artery territory.

More studies assessing the prognostic value and cost-effectiveness are warranted. The current bottleneck which slows the increase of cardiac PET/CT is the limited access to tracers. The clinical value of quantification is under research and in the near future more solid information will become available. The cost-effectiveness of this technique also needs to be studied although additional costs over the standard PET imaging are minimal.

\section{References}

1. Klocke FJ, Baird MG, Lorell BH, Bateman TM, Messer JV, Berman DS, et al. ACC/AHA/ASNC guidelines for the clinical use of cardiac radionuclide imaging - executive summary: A report of the American College of Cardiology/American Heart Association Task Force on Practice Guidelines (ACC/AHA/ASNC Committee to revise the 1995 Guidelines for the Clinical Use of Cardiac Radionuclide Imaging. Circulation 2003;108:1404-18.

2. Dijkmans PA, Knaapen P, Sieswerda GT, Aiazian E, Visser CA, Lammertsma AA, et al. Quantification of myocardial perfusion using intravenous myocardial contrast echocardiography in healthy volunteers: Comparison with positron emission tomography. J Am Soc Echocardiogr 2006;19:285-93.

3. Fritz-Hansen T, Hove JD, Kofoed KF, Kelbaek H, Larsson HB. Quantification of MRI measured myocardial perfusion reserve in healthy humans: A comparison with positron emission tomography. J Magn Reson Imaging 2008;27:818-24.

4. Sampson UK, Dorbala S, Limaye A, Kwong R, Di Carli MF. Diagnostic accuracy of rubidium- 82 myocardial perfusion imaging with hybrid positron emission tomography/computed tomography in the detection of coronary artery disease. J Am Coll Cardiol 2007;49:1052-8.

5. Muzik O, Duvernoy C, Beanlands RS, Sawada S, Dayanikli F, Wolfe ER Jr, et al. Assessment of diagnostic performance of quantitative flow measurements in normal subjects and patients with angiographically documented coronary artery disease by means of nitrogen-13 ammonia and positron emission tomography. J Am Coll Cardiol 1998;31:534-40.

6. Yoshinaga K, Katoh C, Noriyasu K, Iwado Y, Furuyama H, Ito Y, et al. Reduction of coronary flow reserve in areas with and without ischemia on stress perfusion imaging in patients with coronary artery disease: A study using oxygen 15-labeled water PET. J Nucl Cardiol 2003;10:275-83.

7. Parkash R, deKemp RA, Ruddy TD, Kitsikis A, Hart R, Beauchesne $\mathrm{L}$, et al. Potential utility of rubidium 82 PET quantification in patients with 3-vessel coronary artery disease. J Nucl Cardiol 2004;11:440-9.

8. Kajander S, Ukkonen H, Sipilä H, Teräs M, Knuuti J. Low radiation dose imaging of myocardial perfusion and coronary angiography with a hybrid PET/CT scanner. Clin Physiol Funct Imaging 2009;29:81-8.

9. Iida H, Kanno I, Takahashi A, et al. Measurement of absolute myocardial blood flow with $\mathrm{H} 215 \mathrm{O}$ and dynamic positron-emission tomography. Strategy for quantification in relation to the partial-volume effect. Circulation 1988;78:104-15.

10. Hutchins GD, Schwaiger M, Rosenspire KC, et al. Noninvasive quantification of regional blood flow in the human heart using $\mathrm{N}$ 13 ammonia and dynamic positron emission tomographic imaging. J Am Coll Cardiol 1990;15:1032-42.

11. Herrero P, Markham J, Shelton ME, Weinheimer CJ, Bergmann SR. Noninvasive quantification of regional myocardial perfusion PET and myocardial perfusion with rubidium- 82 and positron 
emission tomography. Exploration of a mathematical model. Circulation 1990;82:1377-86.

12. Madar I, Ravert HT, Du Y, Hilton J, Volokh L, Dannals RF, et al. Characterization of uptake of the new PET imaging compound 18 F-fluorobenzyl triphenyl phosphonium in dog myocardium. J Nucl Med 2006;47:1359-66.

13. Huisman MC, Higuchi T, Reder S, Nekolla SG, Poethko T, Wester $\mathrm{HJ}$, et al. Initial characterization of an 18F-labeled myocardial perfusion tracer. J Nucl Med 2008;49:630-6.

14. Plössl K, Chandra R, Qu W, Lieberman BP, Kung MP, Zhou R, et al. A novel gallium bisaminothiolate complex as a myocardial perfusion imaging agent. Nucl Med Biol 2008;35:83-90.

15. Muzik O, Beanlands RS, Hutchins GD, et al. Validation of nitrogen-13-ammonia tracer kinetic model for quantification of myocardial blood flow using PET. J Nucl Med 1993;34:83-91.

16. Bol A, Melin JA, Vanoverschelde JL, et al. Direct comparison of [13 N]ammonia and [15O]water estimates of perfusion with quantification of regional myocardial blood flow by microspheres. Circulation 1993;87:512-25.

17. Lautamäki R, George RT, Kitagawa K, Higuchi T, Merrill J, Voicu $\mathrm{C}$, et al. Rubidium-82 PET-CT for quantitative assessment of myocardial blood flow: Validation in a canine model of coronary artery stenosis. Eur J Nucl Med Mol Imaging 2009;36:576-86.

18. Hesse B, Tägil K, Cuocolo A, Anagnostopoulos C, Bardiés M, Bax $\mathrm{J}$, et al. EANM/ESC procedural guidelines for myocardial perfusion imaging in nuclear cardiology. Eur J Nucl Med Mol Imaging 2005;32:855-97.

19. Gould KL, Pan T, Loghin C, Johnson NP, Guha A, Sdringola S. Frequent diagnostic errors in cardiac PET/CT due to misregistration of CT attenuation and emission PET images: A definitive analysis of causes, consequences, and corrections. J Nucl Med 2007;48:1112-21.

20. Koepfli P, Hany TF, Wyss CA, Namdar M, Burger C, Konstantinidis $\mathrm{AV}$, et al. CT attenuation correction for myocardial perfusion quantification using a PET/CT hybrid scanner. J Nucl Med 2004;45:537-42.

21. Kajander S, Ukkonen H, Joutsiniemi E, Mäki M, Saraste A, AlaKnuuttila E, Pietilä M, Airaksinen J, Hartiala J, Saraste M, Knuuti $\mathrm{J}$. The clinical value of absolute quantification of myocardial perfusion in the detection of coronary artery disease. A study using positron emission tomography to detect multi-vessel disease. Circulation 2008;118:S_1010-S_1011.

22. Anagnostopoulos C, Almonacid A, El Fakhri G, Curillova Z, Sitek A, Roughton M, et al. Quantitative relationship between coronary vasodilator reserve assessed by $82 \mathrm{Rb}$ PET imaging and coronary artery stenosis severity. Eur J Nucl Med Mol Imaging 2008;35: 1593-601.

23. Nesterov SV, Han C, Mäki M, Kajander S, Naum AG, Helenius H, Lisinen I, Ukkonen H, Pietilä M, Joutsiniemi E, Knuuti J. Myocardial perfusion quantitation with 15O-water PET: High reproducibility of the new cardiac analysis software (CarimasTM) Eur J Nucl Med Mol Imaging 2009. [Epub ahead of print].

24. Dorbala S, Vangala D, Sampson U, Limaye A, Kwong R, Di Carli MF. Value of left ventricular ejection fraction reserve in evaluating the magnitude of myocardium at risk and the extent of angiographic coronary artery disease: A $82 \mathrm{Rb}$ PET/CT study. J Nucl Med 2007;48:349-58.

25. Di Carli MF, Hachamovitch R. New technology for noninvasive evaluation of coronary artery disease. Circulation 2007;115:1464-80.

26. Bax JJ, Beanlands RS, Klocke FJ, Knuuti J, Lammertsma AA, Schaefers MA, et al. Diagnostic and clinical perspectives of fusion imaging in cardiology: Is the total greater than the sum of its parts? Heart 2007;93:16-22.

27. O'Neill JO, McCarthy PM, Brunken RC, Buda T, Hoercher K, Young JB, et al. PET abnormalities in patients with nonischemic cardiomyopathy. J Card Fail 2004;10:244-9. 